\title{
An orthogonal terrain-following coordinate and its preliminary tests using 2-D idealized advection experiments
}

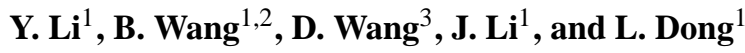 \\ ${ }^{1}$ State Key Laboratory of Numerical Modeling for Atmospheric Sciences and Geophysical Fluid Dynamics, \\ Institute of Atmospheric Physics, Chinese Academy of Sciences, Beijing, China \\ ${ }^{2}$ Ministry of Education Key Laboratory for Earth System Modeling, and Center for Earth System Science, \\ Tsinghua University, Beijing, China \\ ${ }^{3}$ State Key Laboratory of Severe Weather, Chinese Academy of Meteorological Sciences, Beijing, China \\ Correspondence to: Y. Li (liyiyuan@mail.iap.ac.cn)
}

Received: 27 September 2013 - Published in Geosci. Model Dev. Discuss.: 27 November 2013

Revised: 7 July 2014 - Accepted: 11 July 2014 - Published: 25 August 2014

\begin{abstract}
We have designed an orthogonal curvilinear terrain-following coordinate (the orthogonal $\sigma$ coordinate, or the OS coordinate) to reduce the advection errors in the classic $\sigma$ coordinate. First, we rotate the basis vectors of the $z$ coordinate in a specific way in order to obtain the orthogonal, terrain-following basis vectors of the OS coordinate, and then add a rotation parameter $b$ to each rotation angle to create the smoother vertical levels of the OS coordinate with increasing height. Second, we solve the corresponding definition of each OS coordinate through its basis vectors; and then solve the 3-D coordinate surfaces of the OS coordinate numerically, therefore the computational grids created by the OS coordinate are not exactly orthogonal and its orthogonality is dependent on the accuracy of a numerical method. Third, through choosing a proper $b$, we can significantly smooth the vertical levels of the OS coordinate over a steep terrain, and, more importantly, we can create the orthogonal, terrain-following computational grids in the vertical through the orthogonal basis vectors of the OS coordinate, which can reduce the advection errors better than the corresponding hybrid $\sigma$ coordinate. However, the convergence of the grid lines in the OS coordinate over orography restricts the time step and increases the numerical errors. We demonstrate the advantages and the drawbacks of the OS coordinate relative to the hybrid $\sigma$ coordinate using two sets of 2-D linear advection experiments.
\end{abstract}

\section{Introduction}

The complex surface of the Earth is the lower boundary of a numerical atmospheric model, which has become more and more important for operational forecast and scientific research. There are mainly two kinds of methods to deal with the terrain in a model: using a proper vertical coordinate, such as the terrain-following coordinate proposed by Phillips (1957), or using the cut-cell method that has been used in computational fluid dynamics and recently been adapted by many researchers for simulating atmospheric and oceanic flows over irregular geometry (Adcroft et al., 1997; Yamazaki and Satomura, 2010; Lock et al., 2012; Adcroft, 2013; Good et al., 2014; Steppeler et al., 2013). Until now, the terrain-following coordinate has been the most popular choice for the atmospheric and oceanic models: for example, the Hybrid Coordinate Ocean Model (HYCOM; Bleck, 2002; Wallcraft et al., 2009), the Grid-point Atmospheric Model of IAP LASG (GAMIL; Wang et al., 2004), the UK Met Office's Unified Model (MetUM; Davies et al., 2005), the Nucleus for European Modelling of the Ocean (NEMO; Madec, 2008), the Weather Research and Forecasting (WRF; Skamarock et al., 2008) modelling system, the Consortium for Small-scale Modelling (COSMO; Schättler et al., 2012) and the Model for Prediction Across Scales (MPAS; Skamarock et al., 2012).

The terrain-following coordinate ( $\sigma$ coordinate) can be classified into two types: the pressure-based $\sigma$ coordinate originated by Phillips (1957), and the height-based $\sigma$ 
coordinate of Gal-Chen and Somerville (1975). However, both types are non-orthogonal coordinates (Pielke, 2002; Steppeler et al., 2003; Zdunkowski and Bott, 2003; Li et al., 2011). For these $\sigma$ coordinate models, there are significant advection errors and pressure gradient errors near a steep terrain (Smagorinsky et al., 1967; Haney, 1991; Konor and Arakawa, 1997; Ji et al., 2005; Mesinger et al., 2012)

Several methods have been used to smooth the $\sigma$ coordinate levels above a steep terrain to decrease the advection errors. A common method is the hybrid vertical coordinate used in many numerical models (Arakawa and Lamb, 1977; Simmons and Burridge, 1981; Simmons and Strüfing, 1983). More recently, Schär et al. (2002) proposed a new smooth level vertical coordinate (SLEVE), which is a height-based coordinate to smooth the $\sigma$ coordinate levels above a complex terrain, and it has been implemented in the COSMO model. Zängl (2003) extended the SLEVE into a pressurebased coordinate, and Leuenberger (2010) generalized it into a more practicable form. Lately, a smooth terrain-following (STF) coordinate was proposed, which can smooth the $\sigma$ levels much more than the SLEVE and which has been implemented in the MPAS model (Klemp, 2011, 2012). Note that all these methods have been successful at alleviating the advection errors in the $\sigma$ coordinate via smoothing the $\sigma$ coordinate levels above a steep terrain; however, they did not tackle the non-orthogonal basis vectors of the $\sigma$ coordinate.

On the other hand, Thompson et al. (1985) proposed that the more orthogonal the coordinate system is, the smaller a part of the truncation error is. Several methods were proposed to create the 2-D orthogonal, terrain-following grids above a steep terrain in terms of numerical grid generation. Sharman et al. (1988) designed a 2-D orthogonal terrainfollowing grid to increase the numerical accuracy of solving the Poisson equation. Erdun et al. (1997) proposed a conformal mapping technique called Schwarz-Christoffel transformation to generate a 2-D orthogonal grid over complex topography, and this mesh was used in analysing air pollution. However, few efforts were put into a 3-D system or into the design of a 3-D orthogonal, terrain-following coordinate system.

In this study, we aim to reduce the well-known advection errors of the classic $\sigma$ coordinate (CS coordinate) through designing a 3-D orthogonal curvilinear terrain-following coordinate (OS coordinate) in a unique way. First, we take "reversed" steps from those used in designing the CS coordinate: namely, we solve the basis vectors of the new coordinate first and then solve the definition of every coordinate. Based on the basis vectors of the OS coordinate, we solve the three 3-D coordinate surfaces of the OS coordinate. We carry out the 2-D linear advection experiments proposed by Schär (2002) using the OS coordinate and the corresponding hybrid $\sigma$ coordinate to demonstrate the performance of the OS coordinate in reducing the advection errors in the high level above a steep terrain. Finally, we implement the modified experiments by moving the non-zero velocity and the

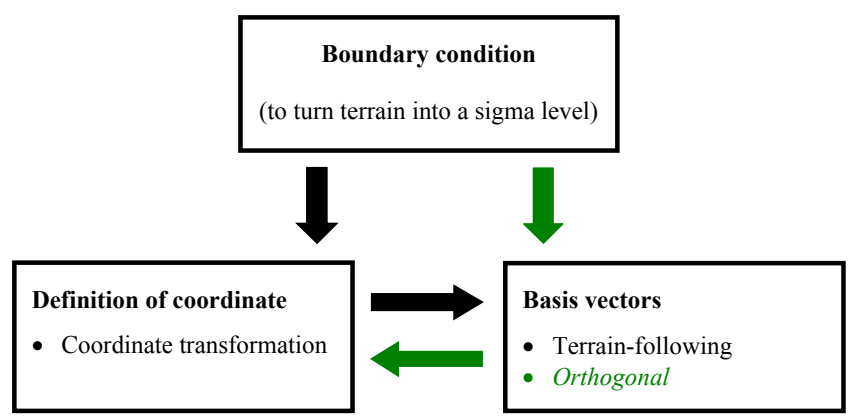

Figure 1. Design of the OS coordinate. The green arrows are for the OS coordinate, and the black arrows, the CS coordinate.

tracer right down to the top of the terrain to investigate the OS coordinate near the mountain top.

\section{The orthogonal curvilinear terrain-following coordinate}

Designing a CS coordinate, researchers first designed the expression of the vertical coordinate in order to turn the terrain into a sigma level, as in Phillips (1957) and Gal-Chen and Somerville (1975), and then solved the basis vectors of the CS coordinate (black arrows in Fig. 1). This design ruined the orthogonality of a coordinate system; however, we reverse these two steps therefore preserving the orthogonality in a $\sigma$ coordinate system (green arrows in Fig. 1).

\subsection{The basis vectors}

We use a unique 3-D coordinate rotation to obtain the basis vectors of the OS coordinate, which is to rotate the basis vectors of the $z$ coordinate until its $z$ axis is in line with the normal vector of the terrain surface (Fig. 2). In particular, we view this 3-D rotation as a combination of two sets of 2-D rotation revolved around the coordinate axes, which are shown as the blue and green arrows in Fig. 2, respectively. Through the two rotation angles $\theta^{\prime}$ and $\lambda^{\prime}$ shown in Fig. 2, we can solve the expressions of these basis vectors, which are orthogonal and terrain following (see Supplement A for the detail).

Moreover, we design a rotation parameter $b$ to make the horizontal basis vectors more horizontal with increasing height and finally equal to the basis vectors of the $z$ coordinate at the top of the model. There are three principles for designing this $b$ : (1) it should be one on the surface of the terrain; (2) it should be zero at the top of the model; and (3) it should monotonically decrease with increasing height. Finally, through adding the rotation parameter $b$ to every rotation angle, we obtain the basis vectors of the OS coordinate (Table 1). 


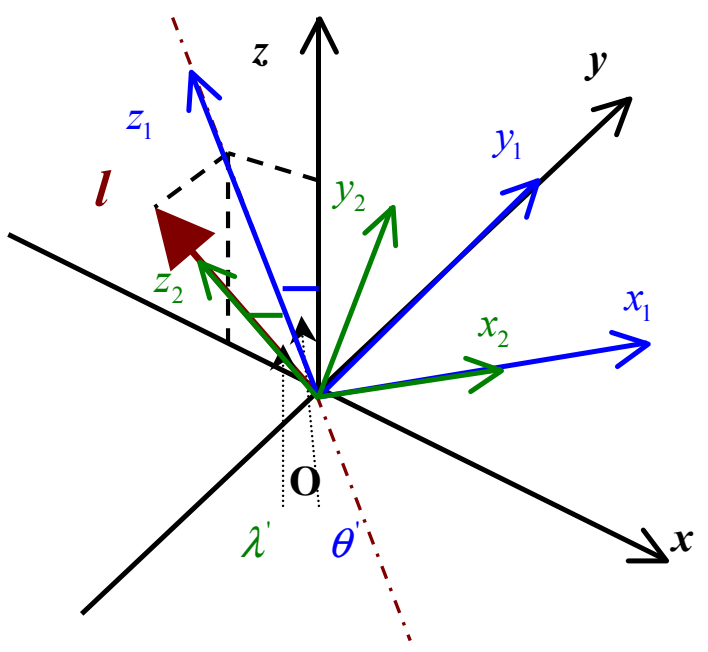

Figure 2. Schematic 3-D rotation for solving the basis vectors of the OS coordinate on the upslope of the terrain. The burgundy arrow is the normal vector of the terrain, and the burgundy dash-dotted line is its projection on the plane $O x z$. The black arrows are the basis vectors of the $z$ coordinate, the blue arrows are the basis vectors of the first rotated coordinate $\left[O ; \boldsymbol{x}_{1}, \boldsymbol{y}_{1}, z_{1}\right]$, and the green arrows are the basis vectors of the second rotated coordinate $\left[O ; \boldsymbol{x}_{2}, \boldsymbol{y}_{2}, \boldsymbol{z}_{2}\right]$.

\subsection{Definition of each coordinate and the 3-D coordinate surfaces}

Through the expression of the basis vectors of the OS coordinate (Table 1), we can obtain the partial differential equations (PDEs) of each OS coordinate as follows (see Supplement B for the detail).

For the horizontal coordinate $x^{\prime}$ of the OS coordinate, we have

$$
\begin{aligned}
& \frac{\partial x^{\prime}}{\partial x}=\cos \left(b \cdot \theta^{\prime}\right), \\
& \frac{\partial x^{\prime}}{\partial y}=0, \\
& \frac{\partial x^{\prime}}{\partial z}=\sin \left(b \cdot \theta^{\prime}\right) .
\end{aligned}
$$

For the horizontal coordinate $y^{\prime}$ of the OS coordinate, we have

$$
\begin{aligned}
& \frac{\partial y^{\prime}}{\partial x}=-\sin \left(b \cdot \theta^{\prime}\right) \cdot \sin \left(b \cdot \lambda^{\prime}\right), \\
& \frac{\partial y^{\prime}}{\partial y}=\cos \left(b \cdot \lambda^{\prime}\right), \\
& \frac{\partial y^{\prime}}{\partial z}=\cos \left(b \cdot \theta^{\prime}\right) \cdot \sin \left(b \cdot \lambda^{\prime}\right) ;
\end{aligned}
$$

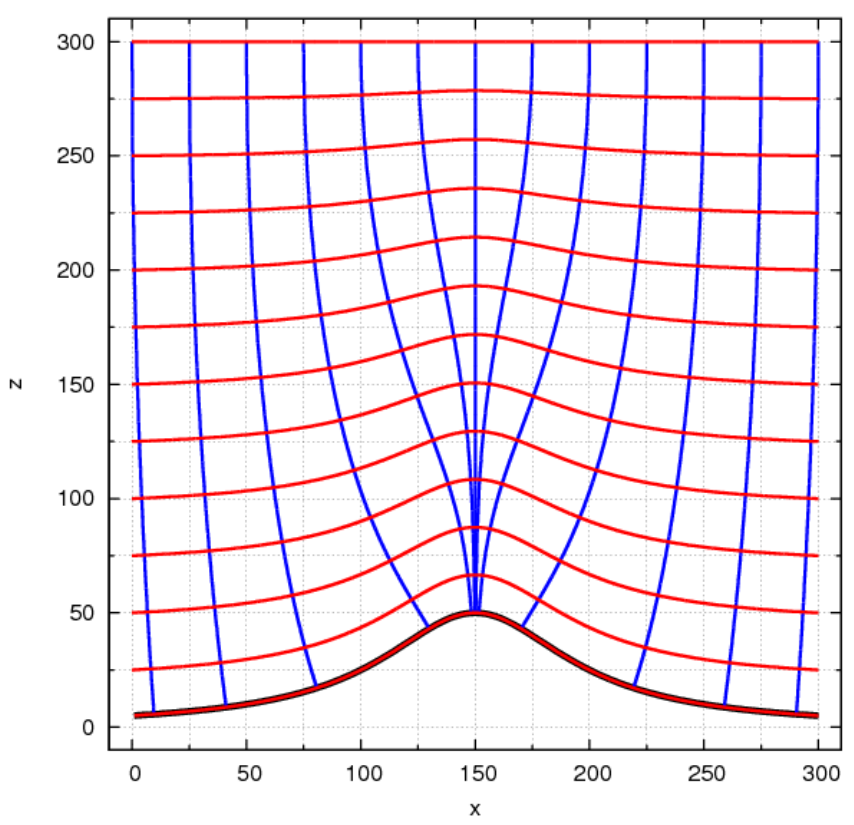

Figure 3. The 2-D grid of the OS coordinate. The red lines are the $\sigma$ levels, the blue lines are the $x^{\prime}$ levels, and the black curve represents the terrain.

with the vertical coordinate $\sigma$ of the OS coordinate,

$$
\begin{aligned}
& \frac{\partial \sigma}{\partial x}=-\sin \left(b \cdot \theta^{\prime}\right) \cdot \cos \left(b \cdot \lambda^{\prime}\right), \\
& \frac{\partial \sigma}{\partial y}=-\sin \left(b \cdot \lambda^{\prime}\right), \\
& \frac{\partial \sigma}{\partial z}=\cos \left(b \cdot \theta^{\prime}\right) \cdot \cos \left(b \cdot \lambda^{\prime}\right) .
\end{aligned}
$$

Solving these three sets of PDEs separately, we can obtain the coordinate transformation between the OS coordinate and the $z$ coordinate (see Supplement B for the detail). The 2-D coordinate lines of the OS coordinate are given in Fig. 3, and that for the 3-D coordinate surfaces is shown in Fig. 4.

Moreover, we examine the orthogonality of the 3-D coordinate surfaces shown in Fig. 4 by calculating the angles between each two normal vectors of the coordinate surfaces (see Supplement C for the detail), and the average of these angles are shown in Fig. 5. Nearly $70 \%$ of angles are in the range of $80-100^{\circ}$ (red in Fig. 5). This can be improved by many methods, such as using high-order numerical methods.

In conclusion, through the orthogonal basis vectors (Table 1) created by the special rotation shown in Fig. 2, the OS coordinate can provide the orthogonal, terrain-following computational grids. Meanwhile, choosing a proper rotation parameter $b$, the OS coordinate can conveniently smooth the vertical levels above a steep terrain (Fig. 3). These are the two benefits of the OS coordinate in reducing the advection errors of the CS coordinate above a steep terrain. 
Table 1. Basis vectors of the OS coordinate.

\begin{tabular}{lr}
\hline Two kinds of basis vectors & Expressions \\
\hline The first kind & $\begin{array}{r}\boldsymbol{i}_{o}=\boldsymbol{i} \cos (b \cdot \lambda)-\boldsymbol{j} \sin (b \cdot \theta) \cdot \sin (b \cdot \lambda)-\boldsymbol{k} \cos (b \cdot \theta) \cdot \sin (b \cdot \lambda) \\
\boldsymbol{j}_{o}=\boldsymbol{j} \cos (b \cdot \theta)-\boldsymbol{k} \sin (b \cdot \theta)\end{array}$ \\
& $\boldsymbol{k}_{o}=\boldsymbol{i} \sin (b \cdot \lambda)+\boldsymbol{j} \sin (b \cdot \theta) \cdot \cos (b \cdot \lambda)+\boldsymbol{k} \cos (b \cdot \theta) \cdot \cos (b \cdot \lambda)$ \\
& $\boldsymbol{i}_{o}=\boldsymbol{i} \cos \left(b \cdot \theta^{\prime}\right)+\boldsymbol{k} \cdot \sin \left(b \cdot \theta^{\prime}\right)$ \\
The second kind & $\boldsymbol{j}_{o}=-\boldsymbol{i} \sin \left(b \cdot \theta^{\prime}\right) \cdot \sin \left(b \cdot \lambda^{\prime}\right)+\boldsymbol{j} \cos \left(b \cdot \lambda^{\prime}\right)+\boldsymbol{k} \cos \left(b \cdot \theta^{\prime}\right) \cdot \sin \left(b \cdot \lambda^{\prime}\right)$ \\
& $\boldsymbol{k}_{o}=-\boldsymbol{i} \sin \left(b \cdot \theta^{\prime}\right) \cdot \cos \left(b \cdot \lambda^{\prime}\right)-\boldsymbol{j} \sin \left(b \cdot \lambda^{\prime}\right)+\boldsymbol{k} \cos \left(b \cdot \theta^{\prime}\right) \cdot \cos \left(b \cdot \lambda^{\prime}\right)$ \\
\hline
\end{tabular}

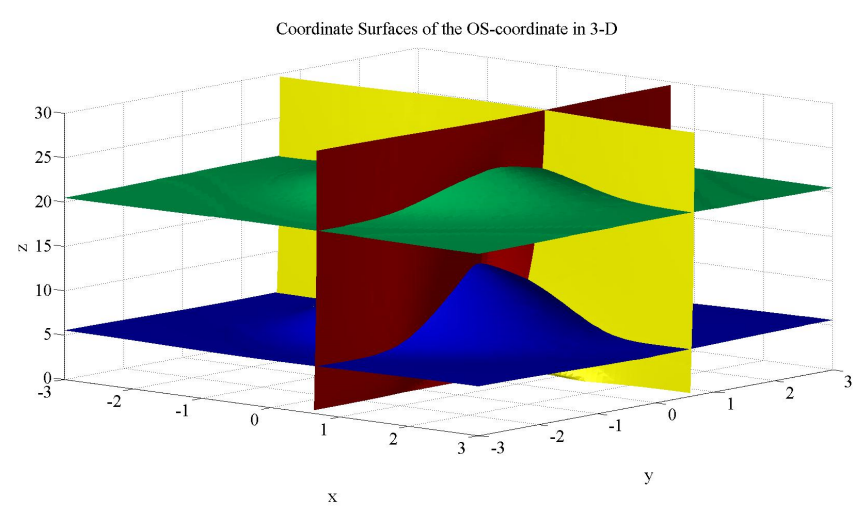

Figure 4. Coordinate surfaces of the OS coordinate in 3-D. The red and yellow surfaces are $x^{\prime}$ and $y^{\prime}$ coordinate surfaces; the blue and green surfaces represent the $\sigma$ coordinate surfaces at two different heights.

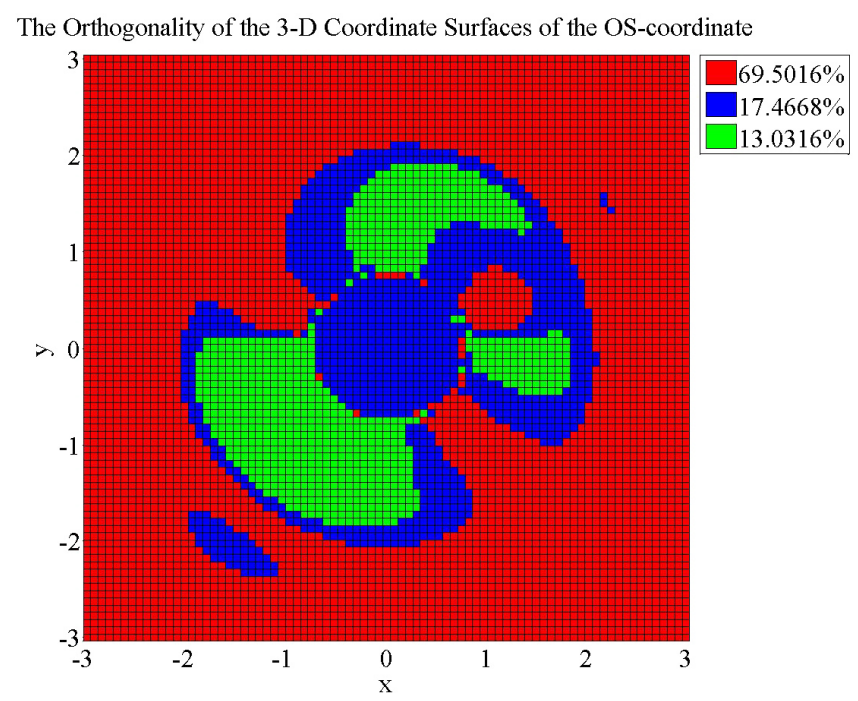

Figure 5. The orthogonality of the points on the coordinate surfaces of the OS coordinate at the same height. Red represents the angle ranges from $80-100^{\circ}$, blue is for $70-80^{\circ}$ or $100-110^{\circ}$, green is for $60-70^{\circ}$ or $110-120^{\circ}$.

\section{Idealized experiments used to compare advection errors}

Since the OS coordinate can smooth the vertical levels and create the orthogonal vertical grids above a steep terrain as shown in Fig. 3, we implement two sets of 2-D linear advection experiments to investigate the distinct effects of the orthogonality and the smoothed levels in reducing the advection errors. First, we reproduce the idealized experiments in Schär et al. (2002) using the OS coordinate and the corresponding hybrid $\sigma$ coordinate (with comparable vertical levels as those in the OS coordinate) to analyse the performance of the OS coordinate in the high-level above a steep terrain. Then, we implement a modified set of experiments by moving the non-zero velocity and the tracer right down to the top of the terrain to further investigate the OS coordinate near the mountain top where the vertical layers are much steeper than those in the high level and also the difference between the OS and hybrid $\sigma$ grids is much greater than that in the high level.

\subsection{Parameters in 2-D linear advection experiments}

For the OS coordinate, we choose the second kind of basis vectors (Table 1) as an example in the following computation, and define its rotation parameter $b=\left(\frac{H_{t}-z}{H_{t}-h}\right)^{n}$. For the CS coordinate, we use the definition of $\sigma=H_{t} \frac{z-h}{H_{t}-h}$, which was proposed by Gal-Chen and Somerville (1975). For the hybrid $\sigma$ coordinate, we use the definition $\sigma=z-$ $\left(\frac{H_{t}-z}{H_{t}-h}\right)^{n} h$, which was proposed by an anonymous reviewer, to create comparable vertical layers as those in the OS coordinate via choosing proper $n$. Note that the vertical layers in the hybrid $\sigma$ coordinate and in the OS coordinate are not exactly equal, due to their different definitions, as we have introduced earlier. This could partly account for the different advection errors in these two coordinates. We will implement more experiments with more comparable vertical layers in the hybrid $\sigma$ coordinate and the OS coordinate in the future.

The expressions of the velocities in these three kinds of coordinates are respectively given as follows. 
For the OS coordinate, we use a non-unit second kind of basis vectors,

$$
\left(\begin{array}{ll}
U & W
\end{array}\right)=\left(\begin{array}{ll}
u & w
\end{array}\right)\left(\begin{array}{ll}
a \cdot \cos \left(b \cdot \theta^{\prime}\right) & -c \cdot \sin \left(b \cdot \theta^{\prime}\right) \\
a \cdot \sin \left(b \cdot \theta^{\prime}\right) & c \cdot \cos \left(b \cdot \theta^{\prime}\right)
\end{array}\right),
$$

where the $a$ and $c$ are two functions which represent the lengths of the horizontal and vertical basis vectors respectively. Here, we estimate their values by the physical grid ( $\Delta x$ and $\Delta z$ ) and the computational grid ( $\Delta X$ and $\Delta Z$ ).

For the CS coordinate, we use the covariant basis vectors,

$$
\left(\begin{array}{ll}
U & W
\end{array}\right)=\left(\begin{array}{ll}
u & w
\end{array}\right)\left(\begin{array}{cc}
1 & H_{t} \cdot \frac{z-H_{t}}{\left(H_{t}-h\right)^{2}} \cdot \frac{\partial h}{\partial x} \\
0 & \frac{H_{t}}{H_{t}-h}
\end{array}\right) ;
$$

and for the hybrid $\sigma$ coordinate, we also use its covariant basis vectors,

$$
\left(\begin{array}{ll}
U & W
\end{array}\right)=\left(\begin{array}{ll}
u & w
\end{array}\right)\left(\begin{array}{cc}
1 & -\left(\frac{H_{t}-z}{H_{t}-h}\right)^{n} \frac{H_{t}+n \cdot h-h}{\left(H_{t}-h\right)} \cdot \frac{\partial h}{\partial x} \\
0 & 1+\frac{n \cdot h}{H_{t}-h} \cdot\left(\frac{H_{t}-z}{H_{t}-h}\right)^{n}
\end{array}\right),
$$

where $u$ and $w$ are the velocities in the $z$ coordinate; $U$ and $W$ are the corresponding velocities in the transformed coordinate.

The advection equation used in all the experiments is the same as in Schär et al. (2002),

$\frac{\partial}{\partial t}\left(J^{-1} q\right)+\frac{\partial}{\partial X}\left(J^{-1} U q\right)+\frac{\partial}{\partial Z}\left(J^{-1} W q\right)=0$,

where $q$ represents the tracer, $U$ and $W$ are the velocities, $J^{-1}$ is the Jacobian of the coordinate transformation, and $X$ and $Z$ are the horizontal and vertical coordinates in each coordinate system. Note that in the CS coordinate the coordinates are solved by the definitions of $X$ and $Z$, while in the OS coordinate the coordinates are calculated following the coordinate transformation illustrated in the Supplement B. There are many schemes to discretize the advection Eq. (13), such as forward, backward and leapfrog schemes of timestepping and spatial discretization schemes of different order. Here, we use the leapfrog time-stepping scheme and the centred spatial discretization scheme. Note that the centred spatial discretization scheme is not suitable for the advection equation, and we just use it as an example. The discretization of Eq. (13) is written as follows:

$$
\begin{aligned}
& \frac{J_{i, k}^{-1}\left(q_{i, k}^{n+1}-q_{i, k}^{n-1}\right)}{2 \Delta t}+ \\
& \frac{J_{i+1, k}^{-1} U_{i+1, k} q_{i+1, k}^{n}-J_{i-1, k}^{-1} U_{i-1, k} q_{i-1, k}^{n}}{2 \Delta X} \\
& +\frac{J_{i, k+1}^{-1} W_{i, k+1} q_{i, k+1}^{n}-J_{i, k-1}^{-1} W_{i, k-1} q_{i, k-1}^{n}}{2 \Delta Z}=0 .
\end{aligned}
$$

The periodic boundary condition is used in the horizontal, while the rigid-lid boundary condition is used in the vertical. The Asselin filter proposed by Asselin (1972), which is commonly used with the leapfrog scheme, is implemented.
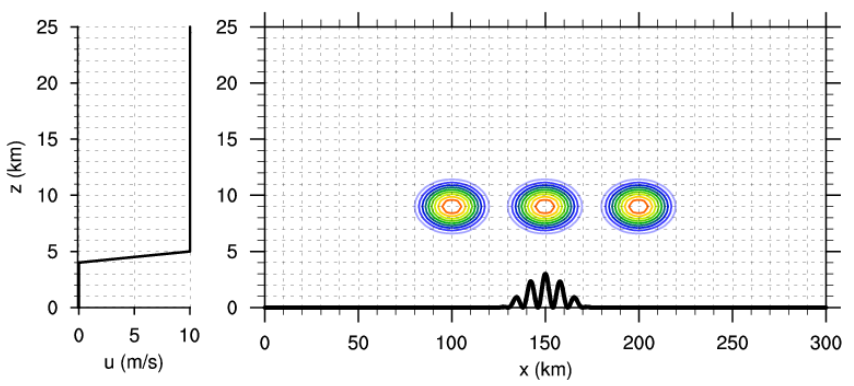

Figure 6. The wind field, the analytical solution of tracer and mountain used in the 2-D linear advection experiments. The left panel is the vertical profile of the analytical solution of the given $u$ field The coloured contours in the right panel represent the tracer $q$ with the contour interval of 0.1 , and the thick black curve, the wavelike terrain.

The analytical solution of tracer $q$ and the wind field of the 2-D advection experiments are shown in Fig. 6. In particular, the domain of the experiments is fixed with $0-300 \mathrm{~km}$ in the horizontal and $0-25 \mathrm{~km}$ in the vertical. And the definitions of the tracer $q$ and the horizontal velocity $u$ follow the advection experiments designed by Schär et al. (2002). The expression of the tracer $q$ is given by

$q(x, z)=q_{0} \cdot\left\{\begin{array}{l}\cos ^{2}\left(\frac{\pi}{2} \cdot r\right), r \leq 1 \\ 0,\end{array}\right.$

where $q_{0}=1, \quad r=\sqrt{\left(\frac{x-x_{0}}{A_{x}}\right)^{2}+\left(\frac{z-z_{0}}{A_{z}}\right)^{2}}, \quad x_{0}=100 \mathrm{~km}$, $A_{x}=25 \mathrm{~km}, A_{z}=3 \mathrm{~km}$, and $z_{0}=9 \mathrm{~km}$ in the high-level experiments. The $u$ field is given by

$u(z)=u_{0} \cdot\left\{\begin{array}{l}1, \quad z_{2} \leq z \\ \sin ^{2}\left(\frac{\pi}{2} \cdot \frac{z-z_{1}}{z_{2}-z_{1}}\right), z_{1} \leq z \leq z_{2} \\ 0, \quad z \leq z_{1},\end{array}\right.$

where $u_{0}=10 \mathrm{~m} \mathrm{~s}^{-1}$, and $z_{1}=4 \mathrm{~km}, z_{2}=5 \mathrm{~km}$ in the highlevel experiments. We also use the wavelike terrain proposed by Schär et al. (2002). The terrain is given by

$h(x)=\cos ^{2}\left(\frac{\pi x}{\lambda}\right) h^{*}(x)$,

where

$h^{*}(x)=\left\{\begin{array}{cc}h_{0} \cos ^{2}\left(\frac{\pi x}{2 a}\right) & \text { for }|x| \leq a \\ 0 & \text { for }|x| \geq a\end{array}\right.$

where $h_{0}$ is the maximum height of the terrain. In all the experiments, we use $h_{0}=3 \mathrm{~km}, a=25 \mathrm{~km}$ and $\lambda=8 \mathrm{~km}$.

In each experiment, the horizontal and vertical resolutions in the computational space are $\mathrm{d} X=1 \mathrm{~km}$ and $\mathrm{d} Z=0.5 \mathrm{~km}$, respectively. We implement five groups of experiments for the three kinds of coordinates - namely, the CS coordinate, two different hybrid editions of the $\sigma$ coordinate $(n=2$ and $24)$, and the OS coordinate with two different $b(n=2$ and 


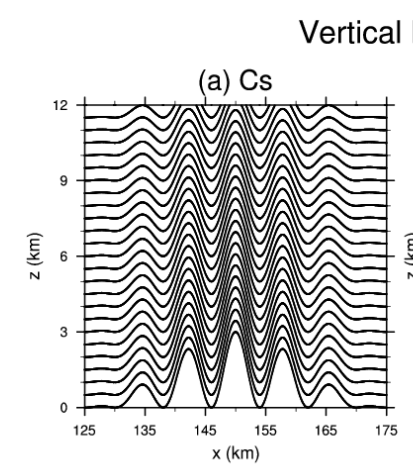

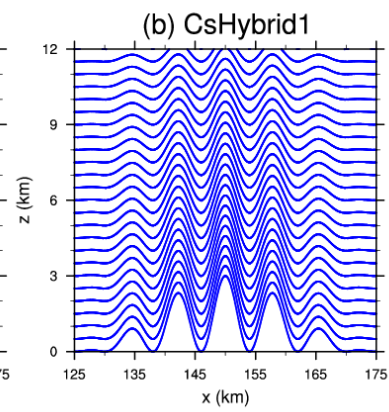

(d) $\mathrm{OsBr} 1$

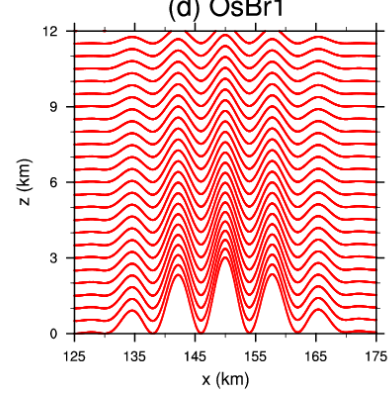

(c) CsHybrid2

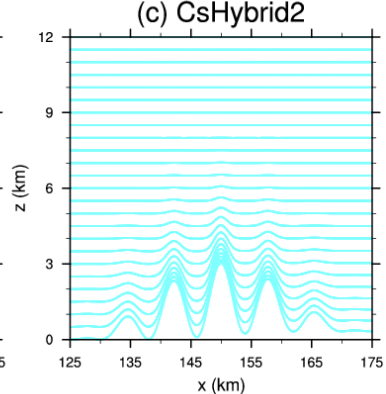

(e) OsBr2

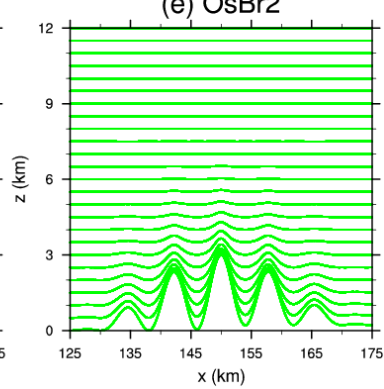

Figure 7. Vertical levels in all the five experiments. The upper three figures are the vertical levels in the CS coordinate, and the two kinds of hybrid $\sigma$ coordinate; the lower two are those in the OS coordinate. Specifically, the black, blue, sky-blue, red and green curves represent the vertical levels in Cs, CsHybrid1, CsHybrid2, OsBr1 and OsBr2 respectively.

20). For simplicity, we abbreviate the five groups of experiments as Cs, CsHybrid1, CsHybrid2, OsBr1 and OsBr2. The vertical levels in these five experiments are shown in Fig. 7; and the OS grid lines in Fig. 7 are solved by the Euler method. The accuracy of grid lines in the OS coordinate can be increased by decreasing the space step of the Euler method, using high-order methods, such as the Runge-Kutta method, or using optimization algorithms to directly solve the PDEs of OS coordinates (see Eqs. 1-9, and Supplement C for the detail).

Figure 8 shows the horizontal and vertical grid lines, which is a close-up of Fig. 7 on the Schär mountain. Note that the skewness is one of the most important qualities of the computational grids for solving the linear advection equation. Skewness is defined to be a normalized distance between a face centre and the intersection of the line joining cell centres with the face. A small value of skewness is beneficial for the accuracy and convergence of the numerical solution (Watton, 2009). Moreover, the skewness in the OS grid (Fig. 8c-d) might be reduced relative to that in the CS grid (Fig. 8a-b), which needs to be carefully investigated in the future. In addition, the centred spatial discretization scheme is not suitable for advection even using a non-skew grid.

The OS grid lines created by power $b$ (Fig. 8c-d) exhibit dramatic convergence and divergence in the horizontal and vertical above mountains and in valleys, which restricts the time step and increases the numerical error. Furthermore, the convergence of the vertical grid lines is due to the terrain-following characteristic of the OS coordinate, which exists in any kind of the terrain-following coordinate, while the convergence of the horizontal grid lines results from the orthogonality of the OS coordinate. So, these convergences and divergences of the grid lines in the OS coordinate cannot be eliminated, but their extent could be alleviated by designing other kind of $b$. Finally, we calculate the horizontal and the vertical Courant numbers of each experiment in the computational space (Table 2). Note that, due to the convergence of the grid lines of the OS coordinate, the time steps used in the OS coordinate are much smaller than those in the CS coordinate. Therefore, designing $b$ properly to control the distance between the grid lines is an essential issue of the OS coordinate, which needs to be carefully investigated in the future.

\subsection{Schär-type (high-level) experiments}

Following Schär et al. (2002), we also calculate the absolute errors (AEs) of the advection in the OS coordinate and the hybrid $\sigma$ coordinate by comparing them with the analytical solution of the advection on every computational grid. First, we compare the AEs of the OS coordinate and the hybrid $\sigma$ coordinate at the end of the integration (Fig. 9), and then we calculate the RMSEs of all the experiments in the whole integration (Fig. 10). The numerical solution of OsBr1 has very large error due to its dramatic convergence of horizontal grid lines shown in Fig. 8c; therefore it cannot be used for a real simulation. Note that the RMSE of $\mathrm{OsBr} 1$ is much larger 
Grid Lines in the Hybrid Sigma-Coordinate and the OS-coordinate

(a) CsHybrid1

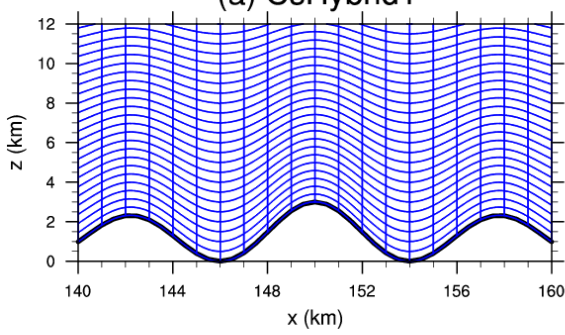

(c) OsBr1

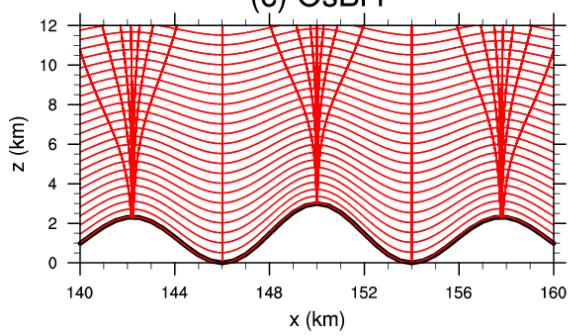

(b) CsHybrid2

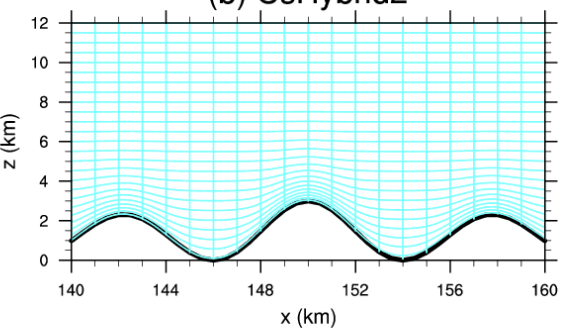

(d) OsBr2

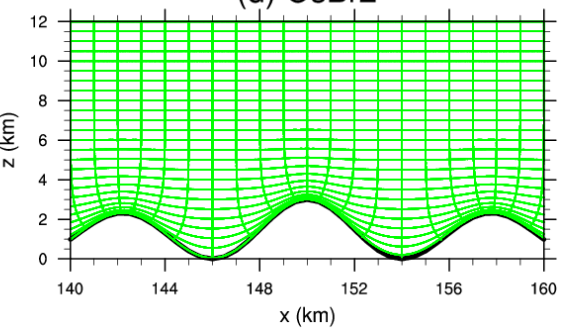

Figure 8. The vertical and horizontal grid lines in the hybrid $\sigma$ coordinate and the OS coordinate.The domain is a close-up of the Schär mountain (i.e. zoomed-in version of Fig. 7). The black curve represents the terrain.

Table 2. Horizontal and vertical Courant numbers of each experiment in the computational space.

\begin{tabular}{lrrrrr}
\hline \multirow{2}{*}{$\begin{array}{c}\text { Experiments } \\
\text { (high and low level) }\end{array}$} & \multicolumn{2}{c}{$\begin{array}{c}\text { Velocity } \\
\text { unit: } \mathrm{m} \mathrm{s}^{-1}\end{array}$} & $\begin{array}{r}\text { Time step } \\
\text { unit: } \mathrm{s}\end{array}$ & \multicolumn{2}{c}{$\begin{array}{c}\text { Maximum } \\
\text { Courant numbers* }\end{array}$} \\
\cline { 2 - 6 } & Maximum $U$ & Maximum $W$ & $\mathrm{~d} t$ & Horizontal & Vertical \\
\hline 1. Cs in high & 10.00 & 10.561 & 25.00 & 0.250 & 0.5281 \\
2. CsHybrid1 in high & 10.00 & 9.567 & 25.00 & 0.250 & 0.4783 \\
3. CsHybrid2 in high & 10.00 & -2.268 & 25.00 & 0.250 & 0.1134 \\
\hline 4. Cs in low & 10.00 & 11.567 & 25.00 & 0.250 & 0.5783 \\
5. CsHybrid1 in low & 10.00 & 11.372 & 25.00 & 0.250 & 0.5686 \\
6. CsHybrid2 in low & 10.00 & -21.823 & 25.00 & 0.200 & 1.0912 \\
\hline 7. OsBr1 in high & 1625.85 & -4.772 & 0.60 & 0.976 & 0.0057 \\
8. OsBr2 in high & 10.65 & 0.795 & 25.00 & 0.266 & 0.0398 \\
\hline 9. OsBr1 in low & 6095.24 & -4.772 & 0.15 & 0.914 & 0.0014 \\
10. OsBr2 in low & 21.00 & -211.932 & 2.00 & 0.042 & 0.8477 \\
\hline
\end{tabular}

* The $\mathrm{d} X=1000 \mathrm{~m}$ and $\mathrm{d} Z=500 \mathrm{~m}$ in the computational space. The Courant numbers are not the critical Courant numbers.

than that of Cs (Fig. 10a), which means using the orthogonal $\sigma$ computational grid with smooth vertical layers can even create a worse solution than using the classic $\sigma$ grid under the condition of dramatic convergence grid lines.

On the other hand, the OsBr2 in which the horizontal grid lines are much sparser than those in OsBr1 (Fig. 8c-d) shows much better solution. However, the AE and the RMSE of $\mathrm{OsBr} 2$ are both equal to those of Cshybrid2 (Fig. 9b and d; Fig. 10b). This is because that in this high-level experiment (above $6 \mathrm{~km}$ ), the vertical layers are very smooth in CsHybrid2 and $\mathrm{OsBr} 2$ (Fig. 7c and e). So the difference between the hybrid $\sigma$ grid and the OS grid is very small above $6 \mathrm{~km}$
(Fig. 8b and d), therefore the difference of the solutions in $\mathrm{OsBr} 2$ and CsHybrid2 is very tiny.

\subsection{Modified Schär-type (low-level) experiments}

In order to further investigate the performance of the OS coordinate near the mountain top, we move the tracer $q$ and the velocity $u$ used in Sect. 3.2 right down to the mountain top by choosing $z_{0}=6 \mathrm{~km}$ in Eq. (15), and $z_{1}=2 \mathrm{~km}$ and $z_{2}=3 \mathrm{~km}$ in Eq. (16). Then we compare the results obtained by the OS coordinate and the hybrid $\sigma$ coordinate. 
Absolute Errors of Both Coordinates in High-Level Experiments
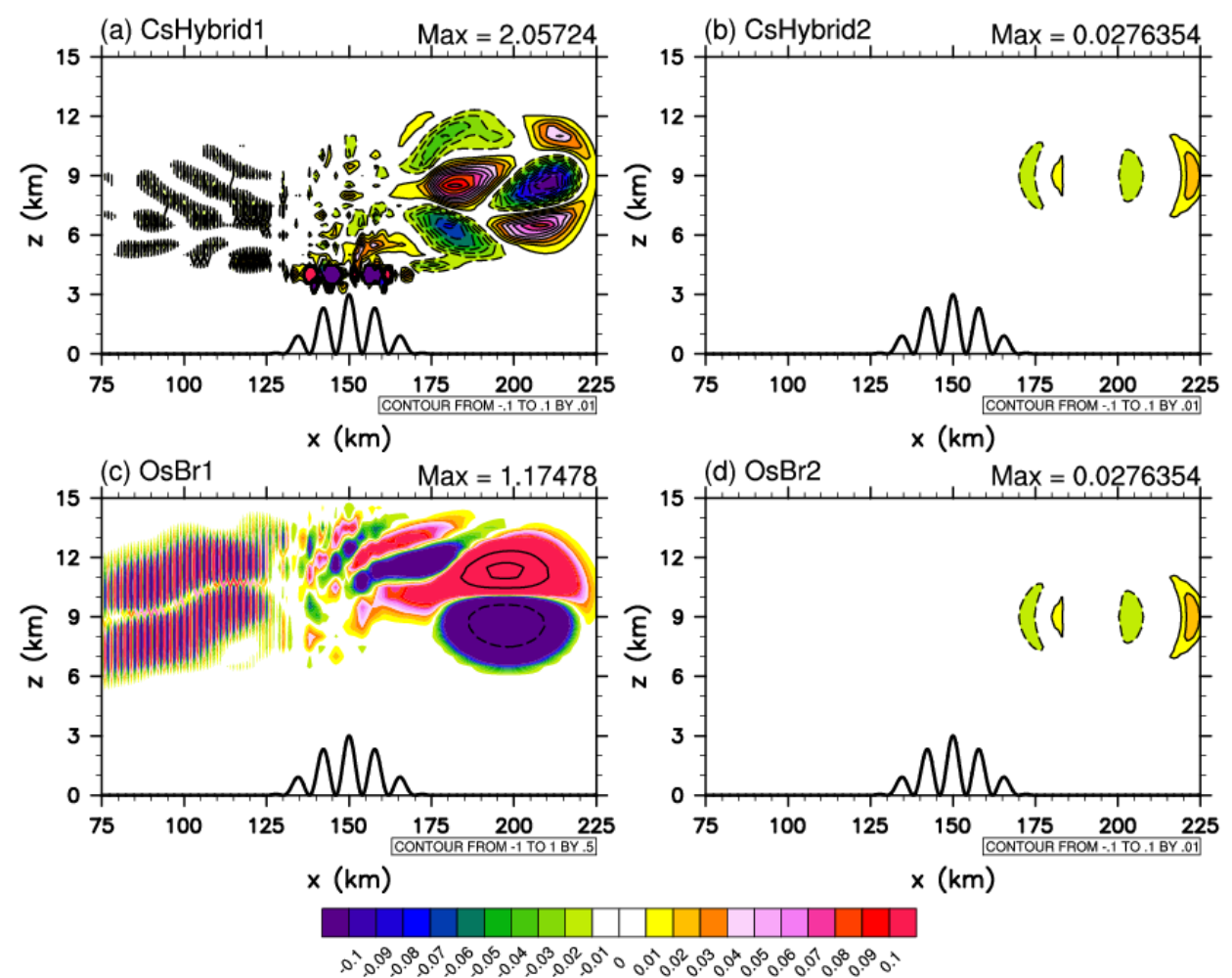

Figure 9. Absolute errors of the hybrid $\sigma$ coordinate and the OS coordinate compared with the analytical solution in the Schär-type (highlevel) experiments at the end of the integration. Shading represents the AE. The solid black contours are for positive values, and the dashed contours are for negative values. The contour interval is 0.01 in (a), (b), and (d); and that in (c) is 0.5 . The solid black curve in the bottom of each figure represents the mountain.

\section{RMSEs of All the Five Coordinates in the High-Level Experiments}
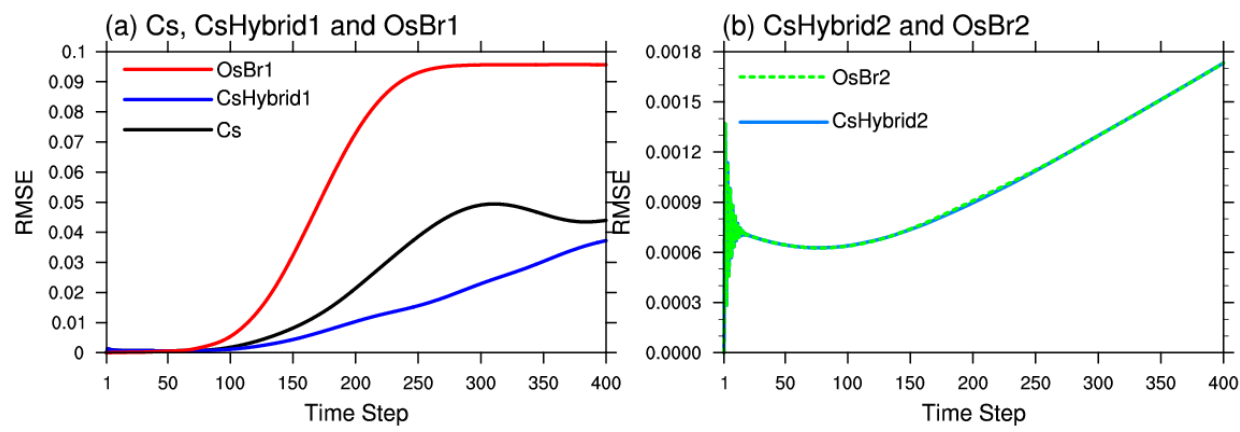

Figure 10. RMSEs of all five experiments with respect to the analytical solution in the Schär-type (high-level) experiments according to the time step calculated by $\mathrm{d} t=25.0$.

First, we compare the results obtained by CsHybrid1, CsHybrid2, OsBr1 and $\mathrm{OsBr} 2$ at three times - namely, at the beginning, the middle and the end of the integration (Fig. 11). The AE of each coordinate at the end of the integration is shown in Fig. 12. Finally, we calculate the RMSEs of all five experiments in the whole integration (Fig. 13). The OsBr1 still shows a bad solution (Fig. 11c). However, comparing with the other three experiments which all have much larger AEs in the low-level experiments (Fig. 12a, b, and d) than those in the high-level experiments (Figs. 9a, $\mathrm{b}$, and $\mathrm{d}$ ), the $\mathrm{AE}$ of $\mathrm{OsBr} 1$ in the low-level experiments (Fig. 12c) is even slightly smaller than that in the high-level experiments (Fig. 9c) - namely this kind of error in OsBr1 may not be dominated by the coordinate transformation error 
Advection of Both Coordinates at Three Times in Low-Level Experiments
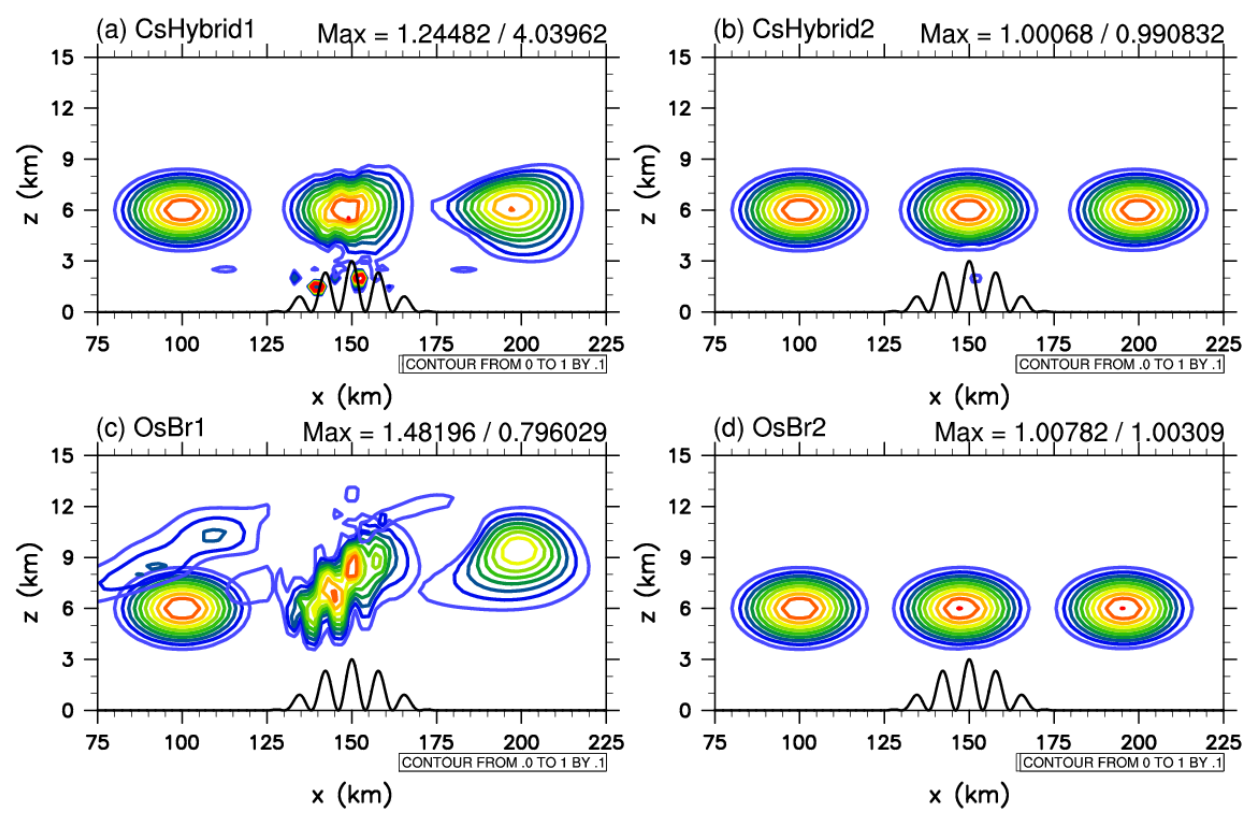

Figure 11. The advection at the beginning, the middle and the end of the integration in the modified Schär-type (low-level) experiments. Coloured contours are the tracer $q$, with the contour interval of 0.1 . The solid black curve in the bottom of each figure represents the mountain.

which has maximum error near the surface of the mountain. On the other hand, we can properly design $b$ to reduce the convergence of the grid lines in OsBr1, and then the errors in OsBr1 might be reduced.

Comparing with the AEs in the high-level experiments (Fig. 9), all the AEs of CsHybrid1, CsHybrid2, OsBr1 and $\mathrm{OsBr} 2$ have extremes near the surface of the mountain in the low-level experiments (Fig. 12). The reason for these extremes needs to be carefully investigated in the future. However, the maximum $\mathrm{AE}$ of $\mathrm{OsBr} 2$ is much reduced relative to that of the CsHybrid2 at the end of the integration (Fig. 12d and b). In particular, the extremes of AE near the mountain surface are all much reduced in the OS coordinate compared with those in the corresponding hybrid $\sigma$ coordinate even in the OsBr1. Furthermore, in the whole integration, the RMSE of the $\mathrm{OsBr} 2$ (green line in Fig. 13b) is much smaller than that of the CsHybrid2 (sky-blue line in Fig. 13b).

Note that the maximum difference of the OS and hybrid $\sigma$ grid is near the surface (Fig. $8 \mathrm{~b}$ and d), and in the low-level experiments the $\mathrm{OsBr} 2$ shows much better performance than that of the CsHybrid2 (Fig. 13b), especially compared with their performances in the high-level experiments (Fig. 10b). This indicates that the more different the computational grid is, the greater the reduction of the advection errors by the OS coordinate is. This needs to be investigated by more experiments near the mountain surface.

\section{Conclusion and discussion}

In order to reduce the "advection errors" in the classic $\sigma$ coordinate, we propose an orthogonal curvilinear terrainfollowing coordinate that has a set of orthogonal and terrainfollowing basis vectors. The key is to use a different approach to re-design a terrain-following coordinate. Since the OS coordinate is orthogonal, the vertical computational grid in the OS coordinate above a steep terrain is orthogonal and terrainfollowing. Moreover, through designing a proper rotation parameter $b$ in the OS coordinate, this new coordinate can conveniently smooth the $\sigma$ levels. Therefore, the OS coordinate can reduce the "advection errors" of the classic $\sigma$ coordinate.

The design of the OS coordinate uses a reversed order from the design of the classic $\sigma$ coordinate, which is to first construct the basis vectors and then the corresponding definition of each coordinate. Specifically, we rotate the basis vectors of the $z$ coordinate until its vertical basis vector is in line with the normal vector of the terrain (Fig. 2), and add a rotation parameter $b$ to each rotation angle (Table 1), which makes the new coordinate orthogonal and terrain following.

To investigate the effect of the OS coordinate in reducing the advection errors, we carried out two sets of 2-D linear advection experiments: the one is the Schär-type (highlevel) experiments, and the other is the modified Schär-type (low-level) experiments; the latter move the tracer and velocity right down to the mountain top. First, in the low-level experiments, the RMSE of OsBr2 experiment (green line in Fig. 13b) is much smaller than that of the corresponding hybrid $\sigma$ coordinate (CsHybrid 2, sky-blue line in Fig. 13b). 


\section{Absolute Errors of Both Coordinates in Low-Level Experiments}
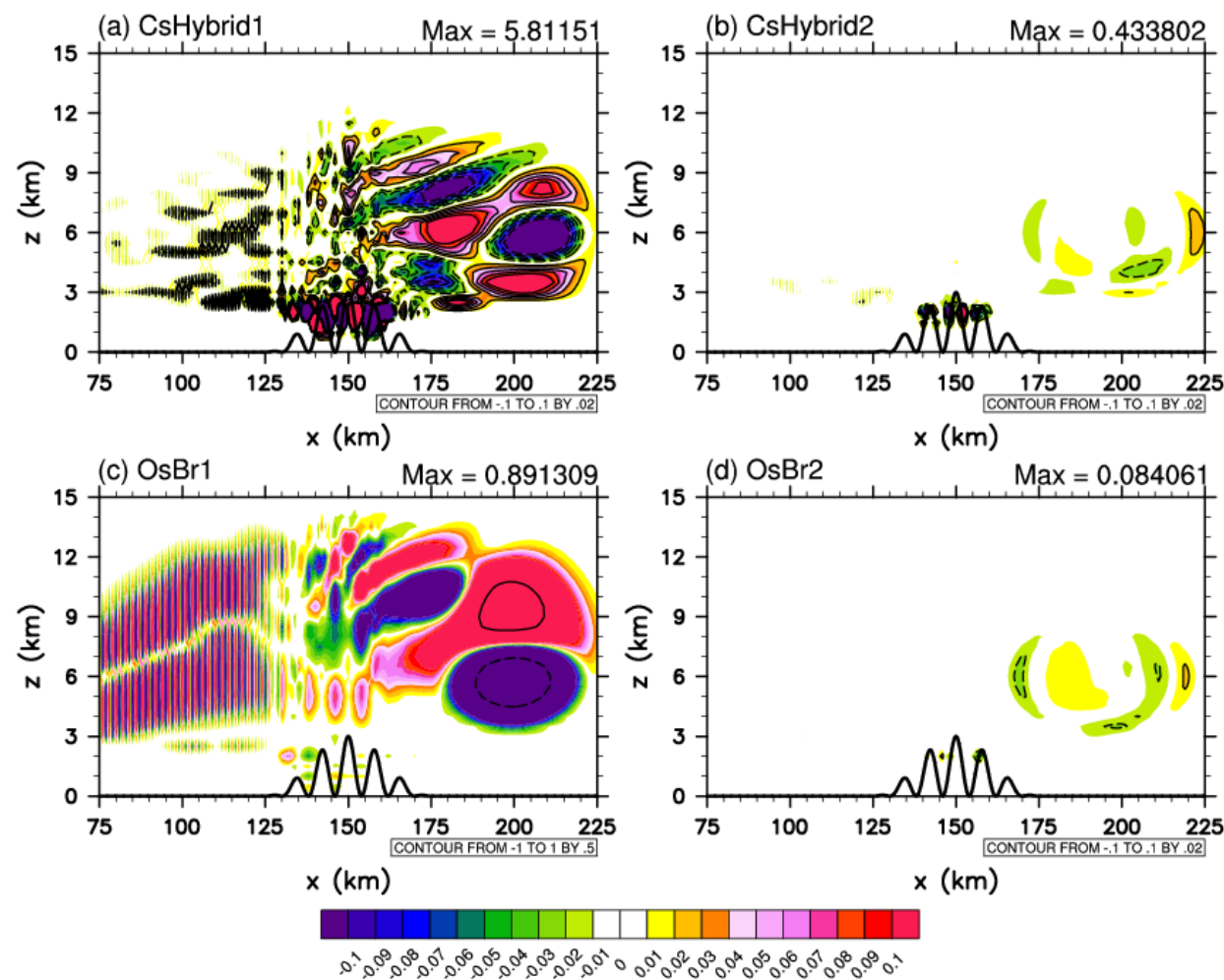

Figure 12. Absolute errors of the hybrid $\sigma$ coordinate and the OS coordinate compared with the analytical solution in the modified Schärtype (low-level) experiments at the end of the integration. Shading represents the AE. The solid black contours are for positive values, and the dashed contours are for negative values. The contour interval is 0.02 in (a), (b), and (d); and that in (c) is 0.5 . The solid black curve in the bottom of each figure represents the mountain. The values of AE under the surface of the mountain are due to the interpolation of plotting.

RMSEs of All the Five Coordinates in the Low-Level Experiments
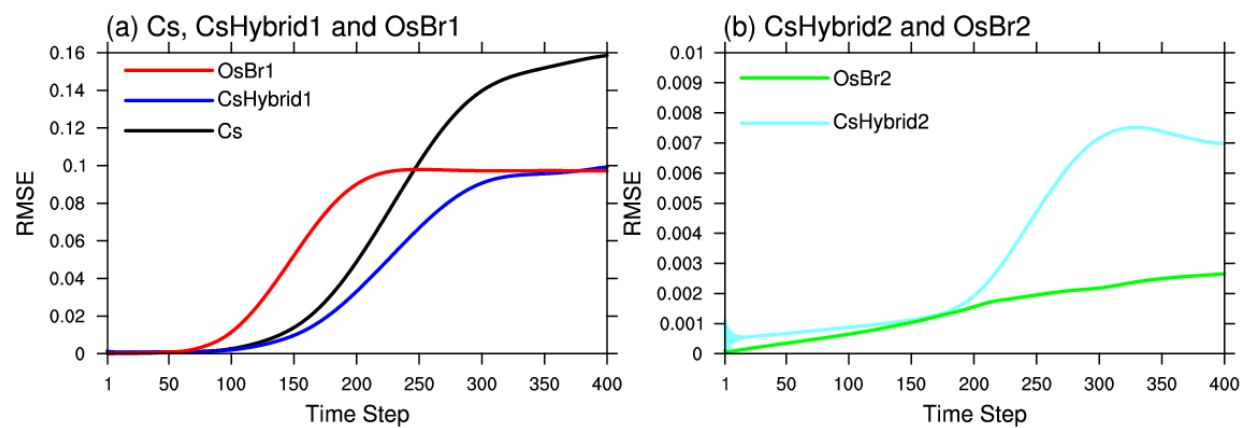

Figure 13. RMSEs of all five experiments with respect to the analytical solution in the modified Schär-type (low-level) experiments according to the time step calculated by $\mathrm{d} t=25.0$.

Second, in the high-level experiments, the RMSE of OsBr2 (green line in Fig. 10b) is equal to that of the CsHybrid2 (deep sky-blue line in Fig. 10b) due to the tiny difference between the OS and the hybrid $\sigma$ grid (Fig. 8b and d). Finally, the OsBr1 experiment shows bad solutions due to the dramatic convergence of the horizontal grid lines (Fig. 8c), which is the major drawback of the OS coordinate. However, this convergence of the OS grid lines could be controlled by properly designing rotation parameter $b$, which is an essential issue of the OS coordinate. In conclusion, the results obtained by the idealized experiments demonstrate that by designing the OS coordinate to create the orthogonal computational grid above a steep terrain, the advection errors can be reduced. Meanwhile, the OS coordinate has major drawbacks 
relative to the non-orthogonal $\sigma$ coordinate over orography because of the convergence and divergence of the grid lines.

In addition, if we use the OS coordinate to solve the momentum equation, the pressure gradient would have just one term in each coordinate direction. It may therefore be possible to calculate the pressure gradient more accurately as noted by Li et al. (2012) and it would be straightforward to ensure that the pressure gradient be curl free in the OS coordinate. This is being tested carefully in an ongoing study. Since the advection errors exist in both atmospheric and oceanic models that use the $\sigma$ coordinate, the OS coordinate can be used for numerical ocean models as well, potentially representing the dynamic effects of bottom topography more precisely and therefore simulating more realistic circulations near complex terrains. The OS coordinate may encounter some problems in practice, such as the CourantFriedrichs-Lewy (CFL) criterion above a steep terrain and the coarse resolution in a narrow valley. Since the computational grid used in this paper is regular, the effect of reduced advection errors by the OS coordinate should be investigated by using irregular grids. Moreover, the OS coordinate should be compared with more other kinds of hybrid $\sigma$ coordinate, such as SLEVE and STF, and the comparison between the OS coordinate with the cut-cell method should be investigated in future in terms of reducing computational errors. The benefits of the new coordinate need to be confirmed by more idealized and realistic experiments.

\section{The Supplement related to this article is available online at doi:10.5194/gmd-7-1767-2014-supplement.}

Acknowledgements. The comments of Hilary Weller (editor) and three anonymous reviewers helped to improve greatly the analyses of advection experiments, the design of the 3-D coordinate surfaces of the OS coordinate and the presentation of the paper. Y. Li wishes to acknowledge helpful discussion about the CFL criterion in the OS coordinate with Hilary Weller, Shiming $\mathrm{Xu}$, and Lijuan Li; she also appreciates discussing the FORTRAN codes of using the hybrid $\sigma$ coordinate in the advection experiments with Jiaqiang Wang. J. Li wishes to acknowledge the help of Shichao Ma and Wentao Cao for optimizing the MATLAB codes of solving the 3-D coordinate surfaces of the OS coordinate. We also appreciate the editing of Zuojun Yu for the presentation of the paper.

Y. Li and B. Wang were supported by the Knowledge Innovation Program of the Chinese Academy of Sciences (KZCX2-YWQ11-04) and the National Basic Research Program of China (973 Program, grant no. 2011CB309704). Y. Li. and J. Li were supported by the National Natural Science Foundation of China (NSFC, grant no. 41305095), and D. Wang, by the NSFC (grant no. 41175064).

Edited by: H. Weller

\section{References}

Adcroft, A.: Representation of topography by porous barriers and objective interpolation of topographic data, Ocean Modell., 67, 13-27, 2013.

Adcroft, A., Hill, C., and Marshall, J.: Representation of topography by shaved cells in a height coordinate ocean model, Mon. Weather Rev., 125, 2293-2315, 1997.

Arakawa, A. and Lamb, V. R.: Computational design of the basic dynamical processes of the UCLA general circulation model, Methods in Computational Physics: Advances in Research and Applications, edited by: Chang, J., Academic Press, 17, 173265, 1977.

Asselin, R.: Frequency filter for time integrations, Mon. Weather Rev., 100, 487-490, 1972.

Bleck, R.: An oceanic general circulation model framed in hybrid isophycnic-Cartesian coordinates, Ocean Modell., 37, 5588, 2002.

Davies, T., Cullen, M. J. P., Malcolm, A. J., Mawson, M. H., Staniforth, A., White, A. A., and Wood, N.: A new dynamical core for the Met Office's global and regional modelling of the atmosphere, Q. J. Roy. Meteor. Soc., 131, 1759-1782, 2005.

Erdun, H.: Two-dimensional atmospheric flow modelling by using Schwarz-Christoffel transformation over complex topography, Ph. D. thesis, ITU, 1997.

Gal-Chen, T. and Somerville, R. C. J.: On the use of a coordinate transformation for the solution of the Navier-Stokes Equations, J. Comput. Phys., 17, 209-228, 1975.

Good, B., Gadian, A., Lock, S.-J., and Ross, A.: Performance of the cut-cell method of representing orography in idealized simulations, Atmos. Sci. Lett., 15, 44-49, 2014.

Haney, R. L.: On the pressure gradient force over steep topography in sigma coordinate ocean models, J. Phys. Oceanogr, 21, 610619, 1991.

Ji, L., Chen, J., Zhang, D., and Wang, H.: Review of some numerical aspects of the dynamic framework of NWP model, Chin. J. Atoms. Sci., 29, 120-130, 2005 (in Chinese).

Klemp, J. B.: A terrain-following coordinate with smoothed coordinate surfaces, Mon. Weather Rev., 139, 2163-2169, 2011.

Klemp, J. B.: Experiences with a smoothed terrain-following vertical coordinate in MPAS, Multiscale numerics for the atmosphere and ocean, Cambridge, UK, 3 October 2012.

Konor, C. S. and Arakawa, A.: Design of an atmospheric model based on a generalized vertical coordinate, Mon. Weather Rev., 125, 1649-1673, 1997.

Leuenberger, D., Koller, M., Fuhrer, O., and Schär, C.: A generelization of the SLEVE vertical coordinate, Mon. Weather Rev., 138, 3683-3689, 2010.

Li, Y., Wang, B., and Wang, D.: Characteristics of terrain-following sigma coordinate, Atmos. Oceanic Sci. Lett., 4, 157-161, 2011.

Li, Y., Wang, D., and Wang, B.: A new approach to implement sigma coordinate in a numerical model, Commun. Comput. Phys., 12, 1033-1050, 2012.

Lock, S.-J., Bitzer, H., Coals, A., Gadian, A., and Mobbs, S.: Demostration of a cut-cell representation of 3-D orography for studies of atmospheric flows over steep hills, Mon. Weather Rev., 140, 411-424, 2012.

Madec, G.: Nemo ocean engine, Note du Pôle de modélisation, Institut Pierre-Simon Laplace(IPSL), France, 27, ISSN No 1288 1619, 2008. 
Mesinger, F., Chou, S.-C., Gomes, J. L., Jovic, D., Bastos, P., Bustamante, J. F., Lazic, L., Lyra, A. A., Morelli, S., Ristic, I., and Veljovic, K.: An upgraded version of the eta model, Meteorol. Atmos. Phys., 116, 63-79, 2012.

Phillips, N. A.: A coordinate system having some special advantages for numerical forecasting, J. Meteorol., 14, 184-185, 1957.

Pielke, R. A.: Mesoscale meteorological modelling, Academic press, 2002.

Schär, C., Leuenberger, D., Fuhrer, O., Lüthi, D., and Girard, C.: A new terrain-following vertical coordinate formulation for atmospheric prediction models, Mon. Weather Rev., 130, 2459-2480, 2002.

Sharman, R. D., Keller, T. L., and Wurtele, M. G.: Incompressible and anelastic flow simulations on numerically generated grids, Mon. Weather Rev., 116, 1124-1136, 1988.

Schättler, U., Doms, G., and Schraff, C.: A description of the nonhydrostatic regional COSMO-model part VII: user's guide, available at: http://www.cosmo-model.org/content/ model/documentation/core/cosmoUserGuide.pdf (last access: 25 September 2013), 2012.

Simmons, A. J. and Burridge, D. M.: An energy and angularmomentum conserving vertical finite-difference scheme and hybrid vertical coordinates, Mon. Weather Rev., 109, 758-766, 1981.

Simmons, A. J. and Strüfing, R.: Numerical forecasts of stratospheric warming events using a model with a hybrid vertical coordinate, Q. J. Roy. Meteor. Soc., 109, 81-111, 1983.

Skamarock, W. C., Klemp, J. B., Dudhia, J., Gill, D. O., Barker, D. M., Duda, M. G., Huang, X-Y., Wang, W., and Powers, J. G.: A description of the advanced research WRF Version 3. NCAR Tech. Note NCAR/TN-475+STR, 113 pp., 2008.

Skamarock, W. C., Klemp, J. B., Duda, M. G., Fowler, L. D., Park, S.-H., and Ringler, T.: A multiscale nonhydrostatic atmospheric model using centroidal Voronoi tesselations and C-grid staggering, Mon. Weather Rev., 140, 3090-3105, 2012.
Smagorinsky, J., Strickler, R. F., Sangster, W. E., Manabe, S., Jr.Halloway, J. L., and Hembree, G. D.: Prediction experiments with a general circulation model, Proc. Int. Symp. on Dynamics of Large Scale Atmospheric Processes, Moscow, USSR, 70-134, 1967.

Steppeler, J., Hess, R., Schattler, U., and Bonaventura, L.: Review of numerical methods for nonhydrostatic weather prediction models, Meteorol. Atmos. Phys., 82, 287-301, 2003.

Steppeler, J., Park, S.-H., and Dobler, A.: Forecasts covering one month using a cut-cell model, Geosci. Model Dev., 6, 875-882, doi:10.5194/gmd-6-875-2013, 2013.

Thompson, J. F., Warsi, Z. U. A., and Mastin, C. W.: Numerical Grid Generation foundations and applications, NorthHolland, 237-249, 1985.

Wallcraft, A. J., Metzger, E. J., and Carroll, S. N.: Software design description for the Hybrid coordinate ocean model (HYCOM) version 2.2. NRL/MR/7320-09-9166, 149 pp., 2009.

Wang, B., Wan, H., Ji, Z., Zhang, X., Yu, R., Yu, Y., and Liu, H.: Design of a new dynamical core for global atmospheric models based on some efficient numerical methods, Sci. China, Ser. A, 47, Supp. 4-21, 2004.

Watton, J.: Fundamentals of fluid power control, Cambridge University press, 2009.

Yamazaki, H. and Satomura, T.: Nonhydrostatic atmospheric modeling using a combined Cartesian grid, Mon. Weather Rev., 138, 3932-3945, 2010.

Zängl, G.: A generalized sigma-coordinate system for the MM5, Mon. Weather Rev., 131, 2875-2884, 2003.

Zdunkowski, W. and Bott, A.: Dynamics of the atmosphere a course in theoretical meteorology, Cambridge University press, 2003. 